ESAIM: COCV

Vol. $13, \mathrm{~N}^{\mathrm{O}} 1,2007$, pp. $163-177$

DOI: $10.1051 /$ cocv:2007006
ESAIM: Control, Optimisation and Calculus of Variations

www.edpsciences.org/cocv

\title{
CONFORMAL MAPPING AND INVERSE CONDUCTIVITY PROBLEM WITH ONE MEASUREMENT
}

\author{
Marc Dambrine and Djalil Kateb ${ }^{1}$
}

\begin{abstract}
This work deals with a two-dimensional inverse problem in the field of tomography. The geometry of an unknown inclusion has to be reconstructed from boundary measurements. In this paper, we extend previous results of $\mathrm{R}$. Kress and his coauthors: the leading idea is to use the conformal mapping function as unknown. We establish an integrodifferential equation that the trace of the Riemann map solves. We write it as a fixed point equation and give conditions for contraction. We conclude with a series of numerical examples illustrating the performance of the method.
\end{abstract}

Mathematics Subject Classification. 49N45, 49Q10, 30C30.

Received April 27, 2005. Revised November 2, 2005.

\section{INTRODUCTION}

In this paper we address the inverse conductivity problem with one measurement: given a bounded, simplyconnected domain $\Omega \subset \mathbb{R}^{2}$, with a smooth boundary and constant conductivity $\sigma_{1}$, we determine from boundary measurements on $\partial \Omega$ an unknown inclusion $\omega \subset \subset \Omega$ whose constant conductivity $\sigma_{2}$ is such that $\sigma_{2} \neq \sigma_{1}$. More precisely, we determine the unknown object $\omega$ in the following Dirichlet problem

$$
\left\{\begin{aligned}
-\operatorname{div}(\sigma \nabla u) & =0 \text { in } \Omega, \\
u & =f \text { on } \partial \Omega, \\
\left\langle\sigma_{1} \nabla u, \nu\right\rangle & =g \text { on } \partial \Omega, \\
\sigma & =\sigma_{1}+\left(\sigma_{2}-\sigma_{1}\right) \chi_{\omega} \text { in } \Omega .
\end{aligned}\right.
$$

Here $\nu$ stands for the unit outer normal to the boundary $\partial \Omega$. We assume in this work that the inclusion $\omega$ is simply-connected. The known data in (1) is the Cauchy pair $(f, g)$. Hereafter, we shall refer to $f$ as Dirichlet data and to $g$ as Neumann data. In practice, it is the inverse problem which determines the inclusion $\omega$ : a voltage $f$ is imposed, and the flux $g=\left\langle\sigma_{1} \nabla u, \nu\right\rangle$ through an accessible outer boundary $\partial \Omega$ is measured. Mathematically, this amounts to knowing the Dirichlet-Neumann map $\Lambda_{\omega}: H^{\frac{1}{2}}(\partial \Omega) \mapsto H^{-\frac{1}{2}}(\partial \Omega)$ defined by $\left.\left.u\right|_{\partial \Omega} \mapsto\left\langle\sigma_{1} \nabla u, \nu\right\rangle\right|_{\partial \Omega}$.

The inverse conductivity problem has attracted much attention because of the large variety of its applications, which include medical imaging and geophysical prospection. In recent years a great deal of attention has been devoted to the uniqueness and stability aspects of the inverse problem from one measurement. Several partial

Keywords and phrases. Inverse conductivity problem, conformal mapping.

${ }^{1}$ Laboratoire de Mathématiques Appliquées de Compiègne. Université de Technologie de Compiègne. Centre de Recherche de Royalieu 60200 Compiègne, France; marc.dambrine@dma.utc.fr; dkateb@utc.fr 
answers have been given for particular inclusions, such as small perturbations of disks, but a general result for uniqueness is still lacking.

The two main difficulties facing any numerical reconstruction method are, first, that this kind of inverse problem is severely ill-posed and, secondly, its nonlinearity. A number of reconstruction algorithms have been proposed, most of which are based on least-square methods or Newton-type iterative schemes. However, the success of these methods depends crucially on a good initial guess. Without this, or without a priori information regarding the inclusion, the method requires considerable additional computational effort : in general this means significantly more iterations. Unfortunately, few numerical algorithms have been proposed in the literature for making a good initial guess.

This work focuses on a numerical algorithm for determining the location and estimating the size of the disk to be used as an initial guess. Our location algorithm, inspired by the work of Kress et al., is based essentially on the theory of conformal mappings and on the special properties of Möbius transforms. Once we have established the initial guess, a standard Newton-type optimization algorithm can be used for reconstructing the shape of the original inclusion.

The paper is organized as follows. In Section 2 we introduce the different objects and results needed for our work. We then consider a first identification algorithm. In Section 3 we describe the motivation of our work and present a rigorous justification of our algorithm in relation to disks. More precisely, we confine ourselves to disks whose centers are not far from the origin. We transform the original problem into a nonlocal and nonlinear integro-differential equation with boundary conditions. We express it as a fixed-point problem for an operator $K$ (see (11) for its precise definition). This fixed point generates an iterative resolution algorithm in the spirit of Akdumann and Kress. In particular, we introduce an auxiliary problem that allows us to generate virtual additional data and to avoid division by the vanishing outgoing flux $\int_{\partial \Omega} g(s) \mathrm{d} s$. In Section 4 we study the convergence of the process. We proceed by linearizing around annuli and carefully selecting the voltage measurement $f$. In the final section we illustrate our theoretical results with some numerical experiments. We show that our algorithm works satisfactorily on domains corresponding to certain $\varepsilon-$ perturbations of a disk. We conclude with some remarks on the limiting cases of our theoretical results.

\section{Notations And Definitions. Preliminary FACts}

\section{Poisson equation in an annulus}

We consider the unit disk $B_{1}$ of boundary $S_{1}$. For $\rho \in(0,1)$, let $B_{\rho}$ be the disk of radius $\rho$ with boundary $S_{\rho}$ and let $A(\rho, 1)$ be the annulus $B_{1} \backslash B_{\rho}$. Let $\sigma_{1}$ and $\sigma_{2}$ be two nonnegative reals; let $\sigma$ be the function defined in $B_{1}$ as $\sigma(x)=\sigma_{2}$ if $|x|<\rho$ and $\sigma_{1}$ else. We consider the Dirichlet-to-Neumann operator $\mathcal{D}_{\rho}$ for the operator $-\operatorname{div}(\sigma \nabla$. $)$ in $B_{1}$ from $H^{1 / 2}\left(S_{1}\right)$ into $H^{-1 / 2}\left(S_{1}\right)$; this depends on $\sigma_{1}, \sigma_{2}, \rho$. For a given function $f \in H^{1 / 2}\left(S_{1}\right)$, we have $\mathcal{D}_{\rho} f=\sigma_{1} \partial_{n} U$ where $U$ is the solution in $H^{1}\left(B_{1}\right)$ of

$$
\left\{\begin{aligned}
-\operatorname{div}(\sigma \nabla u) & =0 \text { in } B_{1} \\
u & =f \text { on } S_{1}
\end{aligned}\right.
$$

Owing to the specific geometry, we are able to solve this problem explicitly using Laurent series. We set $\mu=\left(\sigma_{1}-\sigma_{2}\right) /\left(\sigma_{1}+\sigma_{2}\right) \in(-1,1)$.

Lemma 1. The operator $\mathcal{D}_{\rho}$ is diagonal on the Fourier basis. More precisely, we have the spectral decomposition

$$
\mathcal{D}_{\rho}\left(\frac{1}{\sqrt{\pi}}\left[\begin{array}{c}
\cos k \theta \\
\sin k \theta
\end{array}\right]\right)=\lambda_{k}\left(\frac{1}{\sqrt{\pi}}\left[\begin{array}{c}
\cos k \theta \\
\sin k \theta
\end{array}\right]\right) \text { with } \lambda_{k}(\rho)=\sigma_{1} k \frac{1-\mu \rho^{2 k}}{1+\mu \rho^{2 k}}
$$

Furthermore, the radius $\rho$ can be recovered from the eigenvalues $\lambda_{k}$ by the formula

$$
\forall k \neq 0, \quad \rho=\left|\frac{\sigma_{1} k-\lambda_{k}}{\mu\left(\sigma_{1} k+\lambda_{k}\right)}\right|^{\frac{1}{2 k}}
$$


The following upper bound on the spectral gap of the operator is needed in Section 3.3.

Lemma 2. Assume $\mu<0$ then $0<\lambda_{k+1}(\rho)-\lambda_{k-1}(\rho)<\lambda_{2}(\rho)$.

Proof of Lemma 2. From (3), we have

$$
\lambda_{k+1}(\rho)-\lambda_{k-1}(\rho)=2 \sigma_{1}\left(1-2 \mu \frac{\rho^{2 k+2}}{1+\mu \rho^{2 k+2}}\right)-2 \sigma_{1} \mu(k-1)\left[\frac{\rho^{2 k+2}}{1+\mu \rho^{2 k+2}}-\frac{\rho^{2 k-2}}{1+\mu \rho^{2 k-2}}\right] .
$$

Since $x \mapsto x /(1+\mu x)$ is strictly increasing on $(0,1)$ and $\mu<0$, we have

$$
\lambda_{k+1}(\rho)-\lambda_{k-1}(\rho)<2 \sigma_{1}\left(1-2 \mu \frac{\rho^{2 k+2}}{1+\mu \rho^{2 k+2}}\right) \leq 2 \sigma_{1}\left(1-2 \mu \frac{\rho^{4}}{1+\mu \rho^{4}}\right) \leq \lambda_{2}(\rho) .
$$

\section{Conformal mapping of doubly-connected domains}

According to the theory of conformal mapping of doubly-connected regions, there exist $\rho>0$ and a map $\Psi_{e}$ that maps conformally the domain $\Omega \backslash \bar{\omega}$ onto the annulus $A(\rho, 1)$ (see [6], [4], [7] and [8]). Once $\rho$ is fixed, there exists $\Psi_{i}$ that maps conformally the domain $\omega$ onto the disk $B_{\rho}$. However, in general, $\Psi_{e}$ and $\Psi_{i}$ do not coincide on $\partial \omega$ and the conformal mapping $\Psi_{e}$ cannot be extended into a conformal map defined on the whole $\Omega$. This has an important consequence regarding the application of conformal mapping techniques to the transmission problem. Conformal mappings have the well-known property of preserving harmonicity. In the general case, where two conformal maps coexist for the interior and the exterior, jump conditions on the interface for the transported problem are modified to compensate the change of diffeomorphisms (see [2]), and no simplifications appear with transport. This is why we focus our attention on a class of conformal mappings - Möbius transforms - that transport both $\Omega$ and $\omega$ onto $B_{1}$ and $B_{\rho}$. A Möbius transform is any mapping of the form

$$
T: z \mapsto w=\frac{a z+b}{c z+d}
$$

where $a, b, c, d$ are complex numbers such that $a d-b c \neq 0$. Circles and straight lines are transformed into circles and straight lines. We recall the following well-known result on Möbius transforms.

Lemma 3. A Möbius transform $T$ maps the unit circle onto itself if and only if $T$ is of form

$$
T: z \mapsto w=\mathrm{e}^{i \alpha} \frac{z-a}{1-\bar{a} z}
$$

where $a=a_{1}+i a_{2}$ satisfies $|a|<1$ and where $\alpha$ is an arbitrary real number.

\section{Transformation of the Dirichlet-to-Neumann operator}

We now turn our attention to changes in the Dirichlet-to-Neumann map after domain deformations by Möbius transforms. A conformal mapping defined on the whole domain $\Omega$ will transform the Dirichlet-to-Neuman map for the transmission problem into an operator connected to the Dirichlet-to-Neumann on the model geometry. If $L$ denotes the length of $\partial \Omega$, we introduce $\Gamma=\{\Gamma(t), t \in[0, L)\}$ an L-periodic parameterization of $\partial \Omega$ by the arc length.

From now on we consider $\Phi=\Psi^{-1}$, the conformal mapping transforming the concentric circles onto $\partial \Omega$ and $\partial \omega$. We normalize the mapping $\Phi$ by setting $\Phi(1)=\Gamma(0)$. If the circle $S_{1}$ is parameterized by $S_{1}=\{\theta(t)=$ $\left.e^{i t}, t \in[0,2 \pi]\right\}$, then we can find a strictly monotonous and smooth bijection $\phi$ such that $\Phi \circ \theta=\Gamma \circ \phi ; \phi$ is the main determination of the argument of $\Phi\left(e^{i t}\right)$. The link between the Dirichlet-to-Neumann map $\mathcal{D}_{\rho}$ and the original Dirichlet map $\Lambda_{\omega}$ is given by the following lemma proved in [1]. We change the letter denoting the Dirichlet-to-Neumann map from $\Lambda$ to $\mathcal{D}$, in order to emphasize that the geometry is now an annulus. 
Lemma 4. Let $u$ be a harmonic function in $\Omega \backslash \omega$, and let us set $v=u \circ \Phi$. Then, the chain rule writes

$$
\frac{\partial v}{\partial n}=\frac{\partial u}{\partial \nu} \frac{\mathrm{d} \phi}{\mathrm{d} t} \text { on } C_{1}
$$

\section{LOCATION SEARCH FOR DISKS}

In this section we present two methods, based on the theory of conformal mapping, for identifying a circular inclusion $\omega$.

\subsection{A first algorithm dedicated to circular inclusions}

From now on, we assume for simplicity that $\Omega$ is the unit disk. This is not a restriction: since $\Omega$ is a simply-connected bounded domain, we know from the Riemann mapping theorem that there exists a conformal mapping $\Theta$ mapping $\Omega$ on the unit disk.

Lemma 4 provides a nonstandard differential equation satisfied by the function $\phi$. Applying Lemma 4 to $u$ solution of (1), we get

$$
\mathcal{D}_{\rho}(f \circ \phi)=\frac{\mathrm{d} \phi}{\mathrm{d} t} g \circ \phi \text { on } C_{1} .
$$

Equation (8) is an equation with respect to the unknowns function $\phi$ and real $\rho \in(0,1)$. The boundary condition $\phi(0)=\phi(2 \pi)+L$ has to be added since $\partial \Omega$ is a closed curve of length $L$. This equation is not a classical differential equation: the right-hand side is nonlocal. This is an equation of type $L\left(x, \partial_{x}\right) u=0$ with boundary conditions, $L$ being a pseudo-differential operator. For such an equation, a solution cannot be obtained using classical tools. Very few results are known regarding the existence and uniqueness of solutions, especially when boundary conditions have to be satisfied. However, in our case, the unknown $\phi$ is the trace of a Möbius transform on $S_{1}$. From Lemma 3, it is the main determination of the argument of $\mathrm{e}^{i \alpha}(z-a) /(1-\bar{a} z)$ where $a=a_{1}+i a_{2}$. For convenience, we denote it $\phi_{\alpha, a_{1}, a_{2}}$. Plugging this generic form of the trace of a Möbius transform, we are led to solve the equation (8) in the least-square sense. For numerical convenience, we use the $L^{2}$ norm instead of the $H^{-1 / 2}$ norm. In practice, we look for $\left(\rho, \alpha, a_{2}, a_{3}\right)$ that minimizes

$$
J\left(\rho, \alpha, a_{1}, a_{2}\right)=\left\|\mathcal{D}_{\rho}\left(f \circ \phi_{\alpha, a_{1}, a_{2}}\right)-\frac{\mathrm{d}}{\mathrm{d} t} \phi_{\alpha, a_{1}, a_{2}} g \circ \phi_{\alpha, a_{1}, a_{2}}\right\|_{L^{2}(0,2 \pi)}^{2} .
$$

We assume that $f \in H^{1}(0,2 \pi)$ and $g \in L^{2}(0,2 \pi)$ in order to make sense of the $L^{2}$ norm arising in $J$. Numerical results will be presented in the last section of this work.

We now introduce another method. We follow the outline of Kress et al. [1] and [5] in deriving the integrodifferential equation satisfied by the trace of the conformal mapping on the exterior boundary. The mapping is then sought as the fixed point of an equation.

\subsection{Transformation of the inverse problem into an integro-differential equation}

In their work [1], Akdumen and Kress solve a equation derived from (8) with a fixed-point method in a convenient functional space incorporating the periodicity condition. This strategy naturally provides an iterative and constructive algorithm to solve (8). They do not restrict themselves to circular inclusions. We shall follow the same strategy to obtain a more robust algorithm. We work in the variational spaces for $f$ and $g:$ in order to incorporate the boundary conditions, we perform the change of unknown

$$
\psi(t)=\phi(t)-\frac{L}{2 \pi} t=\phi(t)-t \text { so that } \psi(0)=\psi(2 \pi)=0
$$

We use the operator $V: H^{1}(0,2 \pi) \rightarrow H^{1}(0,2 \pi)$ defined as

$$
V: \quad \psi \mapsto \psi(t)+t
$$


In [1], equation (8) is written in the integral form

$$
\psi(t)=\int_{0}^{t}\left[\frac{\mathcal{D}_{\rho^{*}}(f \circ V \psi)}{g \circ V \psi}(\tau)-\frac{1}{2 \pi} \int_{0}^{2 \pi} \frac{\mathcal{D}_{\rho^{*}}(f \circ V \psi)}{g \circ V \psi}(\chi) \mathrm{d} \chi\right] \mathrm{d} \tau
$$

In the case considered here, the no-flux condition holds and Neumann data $g$ vanishes. To overcome the division by zero, we relax the problem by introducing one couple of virtual additional measurements. We apply Lemma 4 to an adjoint couple of Dirichlet-Neumann data $(F, G)$ satisfying the following compatibility condition: there exists a function $G$ with mean value 0 in $L^{2}(\partial \Omega)$ such that $g^{2}+G^{2} \geq \gamma$ holds for some $\gamma>0$ on $\partial \Omega$. The function $F$ is then taken as the trace on $\partial \Omega$ of a solution to the Neumann problem $-\Delta w=0$ in $\Omega$ with $\partial_{n} w=G$ on $\partial \Omega$.

To ensure the existence of $G$, we assume that the measured Neumann data $g \neq 0$ is a smooth $2 \pi$-periodic function with mean value 0 and with a finite number of zeros. Search $G$ as $G(x)=\sin x-\alpha$ for some $\alpha \in(0, \pi)$. The function $G$ vanishes only in $\alpha$ and $\pi+\alpha$ on $(0,2 \pi)$. Since $\{x, g(x)=0\}$ is finite, then one can find $\alpha$ such that $\alpha$ and $\pi+\alpha$ are not in this set. Let us note the difference in relation to the situation of Lemma 4: the function $G$ is the normal derivative of a harmonic function in the whole domain $\Omega$ and not only in a neighborhood of the boundary. The couple $(F, G)$ does not represent additional measured data, since $F$ and $G$ are not the traces of a function $u$ satisfying (1). However, they can be seen as simulated data for the reference problem without inclusion.

Using Lemma 4, we get

$$
\mathcal{D}_{\rho}[f \circ \phi]=\frac{\mathrm{d} \phi}{\mathrm{d} t} g \circ \phi \text { and } \mathcal{D}_{0}[F \circ \phi]=\frac{\mathrm{d} \phi}{\mathrm{d} t} G \circ \phi
$$

and then the relaxed equation

$$
\frac{\mathrm{d} \phi}{\mathrm{d} t}=\frac{(g \circ \phi) \mathcal{D}_{\rho}[f \circ \phi]+(G \circ \phi) \mathcal{D}_{0}[F \circ \phi]}{\left(g^{2}+G^{2}\right) \circ \phi} .
$$

Let us introduce the operators $U$ and $K$ defined as

$$
\begin{aligned}
& U: H^{1}(0,2 \pi) \quad \rightarrow \quad L^{2}(0,2 \pi) \\
& \psi \mapsto \frac{(g \circ V \psi) \mathcal{D}_{\rho}[f \circ V \psi]+(G \circ V \psi) \mathcal{D}_{0}[F \circ V \psi]}{\left(g^{2}+G^{2}\right) \circ V \psi} \\
& K: \quad H^{1}(0,2 \pi) \quad \rightarrow \quad H^{1}(0,2 \pi) \\
& \psi \mapsto \int_{0}^{t}\left(U \psi(x)-\frac{1}{2 \pi} \int_{0}^{2 \pi} U \psi(\tau) \mathrm{d} \tau\right) \mathrm{d} x .
\end{aligned}
$$

Equation (10) can be written in integral form as the fixed-point problem: find $\psi$ a fixed point of $K$ in $H^{1}(0,2 \pi)$.

\subsection{Contraction properties of $K$}

In this section we address the question: is $K$ a contraction? We are not able to answer this question in its full generality. However, by linearizing around the explicit solution found in Section 2 in the case of the annulus, we obtain stability results around annuli. 


\section{Linearization of the fixed-point operator}

We first perform a linearization of the operator $U$. Hereafter, the letter $D$ denotes a differential. Around the function $\psi$, in the direction of a function $h$, we have, by straightforward computations:

$$
\begin{aligned}
D U & (\psi) . h=\frac{\left(g^{\prime} \circ V \psi\right) \mathcal{D}_{\rho}[f \circ V \psi] h+(g \circ V \psi)\left[D \mathcal{D}_{\rho}[f \circ V \psi]\left(\partial_{\psi} \rho\right) h+\mathcal{D}_{\rho}\left[h\left(f^{\prime} \circ V \psi\right)\right]\right]}{\left(g^{2}+G^{2}\right) \circ V \psi} \\
& +\frac{\left(G^{\prime} \circ V \psi\right) \mathcal{D}_{0}[F \circ V \psi] h+(G \circ V \psi) \mathcal{D}_{0}[h(F \circ V \psi)]}{\left(g^{2}+G^{2}\right) \circ V \psi} \\
& -2 \frac{\left[\left(g^{\prime} \circ V \psi\right)(g \circ V \psi)+\left(G^{\prime} \circ V \psi\right)(G \circ V \psi)\right]\left[(g \circ V \psi) \mathcal{D}_{\rho}[f \circ V \psi]+(G \circ V \psi) \mathcal{D}_{0}[F \circ V \psi]\right] h}{\left(g^{2}+G^{2}\right)^{2} \circ V \psi} .
\end{aligned}
$$

In our case, the conformal mapping which maps the annulus onto itself can be chosen as the identity. Therefore, by the definition of $\phi$, the function $\psi$ corresponding to the solution of (10) is simply 0 (see Sect. 3.2).

One cannot derive precise estimates of this complicated operator for general couples of given data $(f, g)$. The derivative $\partial_{\psi} \rho$ is in general not easy to deal with. However, we can make a judicious choice of measurements $(f, g)$ such that the derivative $\partial_{\psi} \rho$ vanishes. From a mathematical point of view, this is a tight restriction. However, one can deal with it in the light of particular applications: it corresponds to deciding which measurements should be made in order to be able to reconstruct inclusions.

Lemma 5. If the Dirichlet data $f$ is an eigenfunction of the Dirichlet-to-Neumann operator $\mathcal{D}_{\rho}$ then

$$
\left(\partial_{\psi} \rho\right)_{\mid \psi=0}=0 \text {. }
$$

Proof of Lemma 5. Taking from Lemma $1, f(t)=\sin n t / \lambda_{n}(\rho)$ and $g(t)=\sin n t$ we get the expression of the reconstructed radius (4) as

where

$$
\rho(\phi)=\left|\frac{1}{\mu} \frac{a(\phi)-b(\phi)}{a(\phi)+b(\phi)}\right|^{\frac{1}{2 n}}
$$

By differentiation, we obtain

$$
a(\phi)=\sigma_{1} \int_{0}^{2 \pi} g[\phi(t)] \phi^{\prime}(t) \sin (n t) \mathrm{d} t \quad \text { and } \quad b(\phi)=\int_{0}^{2 \pi} f[\phi(t)] \sin (n t) \mathrm{d} t .
$$

$$
\begin{aligned}
D \rho(\phi) . h & =\left|\frac{1}{\mu}\right|^{\frac{1}{n}} \frac{1}{[a(\phi)+b(\phi)]^{2}}\left|\frac{a(\phi)-b(\phi)}{a(\phi)+b(\phi)}\right|^{\frac{1-2 n}{2 n}}[a(\phi) D b(\phi) \cdot h-b(\phi) D a(\phi) \cdot h] \\
D a(\phi) \cdot h & =\sigma_{1} \int_{0}^{2 \pi}\left[g^{\prime}[\phi(t)] h(t) \phi^{\prime}(t) \sin (n t)+g[\phi(t)] h^{\prime}(t) \sin (n t)\right] \mathrm{d} t, \\
D b(\phi) . h & =\int_{0}^{2 \pi} f^{\prime}[\phi(t)] h(t) \sin (n t) \mathrm{d} t .
\end{aligned}
$$

The linearization point $\psi=0$ corresponds to $\phi=I d_{[0,2 \pi]}$. From the choice of the couple $(f, g)$ and for $\phi=I d_{[0,2 \pi]}$, we obtain, following an integration by parts

$$
a(\phi) D b(\phi) . h-b(\phi) D a(\phi) . h=0, \quad \forall h .
$$

Moreover, it is verified that $a(\phi)+b(\phi) \neq 0$. Hence, $D \rho(\phi) . h=0$ for all $h$.

To simplify, we perform the analysis with

$$
f(t)=\frac{\sin t}{\lambda_{1}(\rho)} \text { and } \quad g(t)=\sin t
$$


This choice of low-frequency data is justified by Lemma 1. Hence we can choose the virtual measure as

$$
F(t)=\frac{\cos t}{\lambda_{1}(0)} \text { and } G(t)=\cos t
$$

Note that $\lambda_{1}(0)=1$. Therefore, after straightforward computations, equation (12) reduces to

$$
D U(0) . h=\frac{\sin t}{\lambda_{1}(\rho)} \mathcal{D}_{\rho}[h(t) \cos (t)]-\cos t \mathcal{D}_{0}[h(t) \sin (t)] \text {. }
$$

We need to evaluate the term $\mathcal{D}_{\rho}[h(t) \cos (t)]$. Making use of the diagonal structure of $\mathcal{D}_{\rho}$ in the Fourier basis, we compute this term for $h$ taken among all the individual elements of the Hilbertian basis. For convenience in the following computations, we introduce the Hilbert basis $\left(\mathbf{C}_{k}, \mathbf{S}_{k}\right)_{k>0}$ of $H^{1}(0,2 \pi)$ defined as

$$
\mathbf{C}_{k}(t)=\frac{\cos k t}{k \sqrt{\pi}} \text { and } \mathbf{S}_{k}(t)=\frac{\sin k t}{k \sqrt{\pi}} .
$$

Any $h \in H^{1}(0,2 \pi)$ writes $h=\sum_{n>0} c_{n} \mathbf{C}_{n}+\sum_{n>0} s_{n} \mathbf{S}_{n}$ and $\|h\|_{H^{1}}^{2}=\sum_{n>0}\left(c_{n}^{2}+s_{n}^{2}\right)$. We obtain (we give the main lines of the computations) for $k>2$ :

$$
\begin{aligned}
D U(0) \cdot \cos k t= & \frac{\sin t}{\lambda_{1}(\rho)} \mathcal{D}_{\rho}[\cos k t \cos t]-\frac{\cos t}{\lambda_{1}(0)} \mathcal{D}_{0}[\cos k t \sin t] \\
= & {\left[\frac{\lambda_{k+1}(\rho)}{4 \lambda_{1}(\rho)}-\frac{\lambda_{k+1}(0)}{4 \lambda_{1}(0)}\right] \sin (k+2) t+\left[\frac{\lambda_{k-1}(\rho)-\lambda_{k+1}(\rho)}{4 \lambda_{1}(\rho)}+\frac{\lambda_{k-1}(0)-\lambda_{k+1}(0)}{4 \lambda_{1}(0)}\right] \sin k t } \\
& +\left[\frac{\lambda_{k-1}(0)}{4 \lambda_{1}(0)}-\frac{\lambda_{k-1}(\rho)}{4 \lambda_{1}(\rho)}\right] \sin (k-2) t .
\end{aligned}
$$

Therefore, after integration we have for $k>2$

$$
\begin{gathered}
D K(0) \cdot \cos k t=\frac{1}{4(k+2)}\left[\frac{\lambda_{k+1}(0)}{\lambda_{1}(0)}-\frac{\lambda_{k+1}(\rho)}{\lambda_{1}(\rho)}\right] \cos (k+2) t+\frac{1}{4(k-2)}\left[\frac{\lambda_{k-1}(\rho)}{\lambda_{1}(\rho)}-\frac{\lambda_{k-1}(0)}{\lambda_{1}(0)}\right] \cos (k-2) t \\
+\frac{1}{4 k}\left[\frac{\lambda_{k+1}(\rho)-\lambda_{k-1}(\rho)}{\lambda_{1}(\rho)}+\frac{\lambda_{k+1}(0)-\lambda_{k-1}(0)}{\lambda_{1}(0)}\right] \cos k t
\end{gathered}
$$

We introduce the notation:

We can easily check the useful formulae

$$
\alpha_{k}(\rho)=\frac{\lambda_{k}(\rho)}{\lambda_{1}(\rho)}-\frac{\lambda_{k}(0)}{\lambda_{1}(0)}
$$

$$
\begin{aligned}
\alpha_{k}(\rho) & =\frac{\lambda_{k}(\rho)}{\lambda_{1}(\rho)}-k=\frac{2 k \mu \rho^{2}\left(1-\rho^{2(k-1)}\right)}{\left(1+\mu \rho^{2 k}\right)\left(1-\mu \rho^{2}\right)}, \\
2+\alpha_{k+1}(\rho)-\alpha_{k-1}(\rho) & =\frac{\lambda_{k+1}(\rho)-\lambda_{k-1}(\rho)}{\lambda_{1}(\rho)} .
\end{aligned}
$$

We perform the same computation for $h=\mathbf{S}_{k}$. Using (17), we get

$$
D K(0) .\left[\begin{array}{c}
\mathbf{C}_{k} \\
\mathbf{S}_{k}
\end{array}\right]=\frac{\alpha_{k-1}(\rho)}{4 k}\left[\begin{array}{c}
\mathbf{C}_{k-2} \\
\mathbf{S}_{k-2}
\end{array}\right]+\frac{4+\alpha_{k+1}(\rho)-\alpha_{k-1}(\rho)}{4 k}\left[\begin{array}{c}
\mathbf{C}_{k} \\
\mathbf{S}_{k}
\end{array}\right]-\frac{\alpha_{k+1}}{4 k}\left[\begin{array}{c}
\mathbf{C}_{k+2} \\
\mathbf{S}_{k+2}
\end{array}\right],
$$


with the initialization:

$$
\begin{aligned}
& D K(0) .\left[\begin{array}{l}
\mathbf{C}_{1} \\
\mathbf{S}_{1}
\end{array}\right]=\frac{4+\alpha_{2}(\rho)}{4}\left[\begin{array}{l}
\mathbf{C}_{1} \\
\mathbf{S}_{1}
\end{array}\right]-\frac{\alpha_{2}(\rho)}{4}\left[\begin{array}{l}
\mathbf{C}_{3} \\
\mathbf{S}_{3}
\end{array}\right], \\
& D K(0) .\left[\begin{array}{l}
\mathbf{C}_{2} \\
\mathbf{S}_{2}
\end{array}\right]=\frac{4+\alpha_{3}(\rho)}{4}\left[\begin{array}{l}
\mathbf{C}_{2} \\
\mathbf{S}_{2}
\end{array}\right]-\frac{\alpha_{3}(\rho)}{4}\left[\begin{array}{l}
\mathbf{C}_{4} \\
\mathbf{S}_{4}
\end{array}\right] .
\end{aligned}
$$

As easily seen in equation (18), the operator $D K(0)$ is almost diagonalized by the Fourier basis. Let us show that it is a contraction.

\section{The contraction property}

We compute $\|D K(0) \cdot h\|_{H^{1}}$ for an arbitrary $h \in H^{1}(0,2 \pi)$ and compare it with the norm of $h$. To improve readability, we drop the dependency of $\alpha$ with respect to $\rho$, since no confusion is possible.

First of all, we remark that if $\mu>0$ then $\left\|D K(0) \cdot \mathbf{C}_{1}\right\|_{H^{1}}>1=\left\|\mathbf{C}_{1}\right\|_{H^{1}}$. That is why, from now on, we assume that $\mu<0$. This assumption means $\sigma_{2}>\sigma_{1}$ : the conductivity is greater inside the inclusion than outside.

We introduce some reduced parameters:

$$
\tau=-\mu \rho^{2} \text { and } \theta=\frac{\tau}{1+\tau} .
$$

Since $\mu<0$, we have $\tau \in(0,1)$ and $\theta \in\left(0, \frac{1}{2}\right)$. We emphasize the fact that $\tau \neq 1$. In the rest of this section, we use the convention that any coefficient with a negative index is 0 . The study of the contraction properties of $D K(0)$ requires a precise study of the $\left(\alpha_{k}\right)$ defined in (16). From the expression of the eigenvalues $\lambda_{k}$ given in (3), we get:

$$
\alpha_{k}(\rho)=2 k \frac{-\tau}{(1+\tau)} \frac{1-\rho^{2(k-1)}}{1-\tau \rho^{2(k-1)}}=-2 k \theta+2 k(1-\tau) \theta \frac{\rho^{2 k-2}}{1-\tau \rho^{2 k-2}} .
$$

In particular, we have the rough estimate

$$
\forall k>0, \quad 0>\alpha_{k}(\rho)>-2 k \theta
$$

Proposition 1. For $h=\sum_{n>0} c_{n} \mathbf{C}_{n}+\sum_{n>0} s_{n} \mathbf{S}_{n}$, one has

$$
\|D K(0) \cdot h\|_{H^{1}}^{2}=A(h)+B(h)+C(h),
$$

where we set

$$
\begin{aligned}
A(h) & =\frac{1}{16} \sum_{k>0} \frac{\left[4+\alpha_{k+1}-\alpha_{k-1}\right]^{2}+\alpha_{k+1}^{2}+\alpha_{k-1}^{2}}{k^{2}}\left(c_{k}^{2}+s_{k}^{2}\right), \\
B(h) & =\frac{1}{8} \sum_{k>0} \frac{\alpha_{k+1}\left(\alpha_{k-1}-2 \alpha_{k+1}+\alpha_{k+3}\right)}{k(k+2)}\left(c_{k+2} c_{k}+s_{k+2} s_{k}\right), \\
C(h) & =-\frac{1}{8} \sum_{k>0} \frac{\alpha_{k+3} \alpha_{k+1}}{k(k+4)}\left(c_{k} c_{k+4}+s_{k} s_{k+4}\right) .
\end{aligned}
$$


Proof of Proposition 1. We apply (18), to $h=\sum_{k>0} c_{k} \mathbf{C}_{k}+s_{k} \mathbf{S}_{k}$. After collecting all the terms of order $k$, we obtain, from Parseval's formula :

$$
\begin{aligned}
\|D K(0) . h\|_{H^{1}}^{2}= & \frac{1}{16} \sum_{k>0}\left[\frac{4+\alpha_{k+1}-\alpha_{k-1}}{k} c_{k}-\frac{\alpha_{k+1}}{k+2} c_{k+2}+\frac{\alpha_{k-1}}{k-2} c_{k-2}\right]^{2} \\
& +\frac{1}{16} \sum_{k>0}\left[\frac{4+\alpha_{k+1}-\alpha_{k-1}}{k} s_{k}-\frac{\alpha_{k+1}}{k+2} s_{k+2}+\frac{\alpha_{k-1}}{k-2} s_{k-2}\right]^{2} .
\end{aligned}
$$

Expanding this expression, we obtain (23) up to some changes of index left to the reader.

\section{Proposition 2. We have}

$$
\begin{gathered}
|A(h)| \leq \frac{1}{8}\left[4+\left(\frac{\lambda_{2}(\rho)}{\lambda_{1}(\rho)}\right)^{2}\right]\|h\|_{H^{1}}^{2} . \\
|B(h)| \leq \frac{4}{3} \theta^{2} \frac{1-\rho^{4}}{1-\tau}\|h\|_{H^{1}}^{2} . \\
|C(h)| \leq \frac{4}{5} \theta^{2}\|h\|_{H^{1}}^{2} .
\end{gathered}
$$

Proof of Proposition 2. We prove (27). For all $k>0$, we set

$$
a_{k}=\frac{\left[4+\alpha_{k+1}+\alpha_{k-1}\right]^{2}+\alpha_{k+1}^{2}+\alpha_{k-1}^{2}}{16 k^{2}}
$$

For $k=1$, we have

$$
a_{1}=\frac{\left(4+\alpha_{2}\right)^{2}+\alpha_{2}^{2}}{16}=\frac{1}{16}\left[\left(\frac{\lambda_{2}(\rho)}{\lambda_{1}(\rho)}-2\right)^{2}+\left(\frac{\lambda_{2}(\rho)}{\lambda_{1}(\rho)}+2\right)^{2}\right]=\frac{1}{8}\left[4+\left(\frac{\lambda_{2}(\rho)}{\lambda_{1}(\rho)}\right)^{2}\right] .
$$

It suffices to prove that $a_{k}<a_{1}, \forall k>1$. First using (22) (a product of two $\alpha_{k}$ is nonnegative), and then (17), we notice that for any $k \geq 2$

$$
\begin{aligned}
a_{k} & =\frac{1}{16 k^{2}}\left[\left(4+\alpha_{k+1}-\alpha_{k-1}\right)^{2}+\left(\alpha_{k+1}-\alpha_{k-1}\right)^{2}-2 \alpha_{k-1} \alpha_{k+1}\right], \\
& \leq \frac{1}{8 k^{2}}\left[4+\left(2+\alpha_{k+1}-\alpha_{k-1}\right)^{2}\right]=\frac{1}{8 k^{2}}\left[4+\left(\frac{\lambda_{k+1}(\rho)-\lambda_{k-1}(\rho)}{\lambda_{1}(\rho)}\right)^{2}\right] .
\end{aligned}
$$

Using the spectral gap estimate stated in Lemma 2, we obtain the upper bound for $k \geq 2$ :

$$
a_{k} \leq \frac{1}{8 k^{2}}\left[4+\left(\frac{\lambda_{2}(\rho)}{\lambda_{1}(\rho)}\right)^{2}\right]<\frac{1}{8}\left[4+\left(\frac{\lambda_{2}(\rho)}{\lambda_{1}(\rho)}\right)^{2}\right]=a_{1}
$$

We turn to (28). First, we consider the case where $k>1$, since we can make use of the structure of a second-order difference. We use (21) to get

$$
\left|\alpha_{k+3}-2 \alpha_{k+1}+\alpha_{k-1}\right|=2 \theta(1-\tau)\left[(k-1) \frac{\rho^{2 k-4}}{1-\tau \rho^{2 k-4}}-2(k+1) \frac{\rho^{2 k}}{1-\tau \rho^{2 k}}+(k+3) \frac{\rho^{2 k+4}}{1-\tau \rho^{2 k+4}}\right] .
$$


We introduce the auxiliary function $a(x)=x /(1-\tau x)$ with derivative $a^{\prime}(x)=1 /(1-\tau x)^{2}>0$. Hence, we have for $t \in(0,1), a(t) \in(0,1 /(1-\tau))$. We apply the mean value theorem: there exist $x_{k} \in\left[\rho^{2 k+4}, \rho^{2 k}\right]$ and $y_{k} \in\left(\rho^{2 k+4}, \rho^{2 k}\right)$ such that

$$
\begin{aligned}
& (k-1) a\left(\rho^{2 k-4}\right)-2(k+1) a\left(\rho^{2 k}\right)+(k+3) a\left(\rho^{2 k+4}\right) \\
& \quad=[(k+3)-2(k+1)+(k-1)] a\left(\rho^{2 k}\right)-(k+3) \rho^{2 k}\left(1-\rho^{4}\right) a^{\prime}\left(x_{k}\right)+(k-1) \rho^{2 k-4}\left(1-\rho^{4}\right) a^{\prime}\left(y_{k}\right) .
\end{aligned}
$$

We get

$$
\begin{aligned}
\mid(k-1) & a\left(\rho^{2 k-4}\right)-2(k+1) a\left(\rho^{2 k}\right)+(k+3) a\left(\rho^{2 k+4}\right) \mid \\
& \leq(k-1)\left|\rho^{2 k-4}-\rho^{2 k}\right| \sup _{t \in\left[\rho^{2 k}, \rho^{2 k-4}\right]} a^{\prime}(t)+(k+3)\left|\rho^{2 k+4}-\rho^{2 k}\right| \sup _{t \in\left[\rho^{2 k+4}, \rho^{2 k}\right]} a^{\prime}(t), \\
& \leq\left(1-\rho^{4}\right)\left[(k-1) \frac{\rho^{2 k-4}}{\left(1-\tau \rho^{2 k-4}\right)^{2}}+(k+3) \frac{\rho^{2 k}}{\left(1-\tau \rho^{2 k}\right)^{2}}\right], \\
& \leq 2(k+1)\left(1-\rho^{4}\right) \frac{\rho^{2 k-4}}{\left(1-\tau \rho^{2 k-4}\right)^{2}} \leq \frac{2(k+1)\left(1-\rho^{4}\right)}{(1-\tau)^{2}} .
\end{aligned}
$$

Hence, we obtain

$$
\left|\alpha_{k+3}-2 \alpha_{k+1}+\alpha_{k-1}\right| \leq 4(k+1) \frac{1-\rho^{4}}{1-\tau} \theta,
$$

from which we deduce with the help of $(22)$

$$
\left|\frac{\left(\alpha_{k-1}-2 \alpha_{k+1}+\alpha_{k+3}\right) \alpha_{k+1}}{8 k(k+2)}\right| \leq \frac{(k+1)^{2}}{k(k+2)} \frac{1-\rho^{4}}{1-\tau} \theta^{2} \leq \frac{4}{3} \frac{1-\rho^{4}}{1-\tau} \theta^{2} .
$$

For $k=1$, we check that

$$
\alpha_{4}-2 \alpha_{2}=8 \theta\left(\rho^{4}-1\right) \frac{\rho^{2}}{1-\tau \rho^{2}} \frac{1-\tau}{1-\tau \rho^{6}} \Rightarrow\left|\frac{\left(\alpha_{4}-2 \alpha_{2}\right) \alpha_{3}}{24}\right| \leq \frac{4}{3} \frac{1-\rho^{4}}{1-\tau} \theta^{2} .
$$

Cauchy-Schwarz inequality brings us to our conclusion.

We now consider the estimation of $C$. For all $k>2$, we deduce from (21)

$$
\left|\frac{\alpha_{k+1} \alpha_{k-1}}{8(k-2)(k+2)}\right| \leq \frac{1}{2} \frac{k+1}{k+2} \frac{k-1}{k-2} \theta^{2} \leq \frac{1}{2} \frac{k^{2}-1}{k^{2}-4} \theta^{2} \leq \frac{4}{5} \theta^{2} .
$$

From this uniform upper bound and Cauchy-Schwarz inequality, we get the upper bound for $C$.

We are now in a position to state the main result of this section.

Theorem 1. If the couple $(\rho, \mu)$ satisfies the condition:

$$
\frac{-\mu \rho^{2}\left(1-\rho^{2}\right)^{2}}{\left(1-\mu \rho^{2}\right)\left(1+\mu \rho^{4}\right)^{2}}-\frac{4 \mu \rho^{2}}{1-\mu \rho^{2}}\left[\frac{1}{5}+\frac{1}{3} \frac{1-\rho^{2}}{1+\mu \rho^{2}}\right]<\frac{1-\rho^{2}}{1+\mu \rho^{4}}
$$

then the operator $D K(0)$ is a contraction on $H^{1}(0,2 \pi)$. 


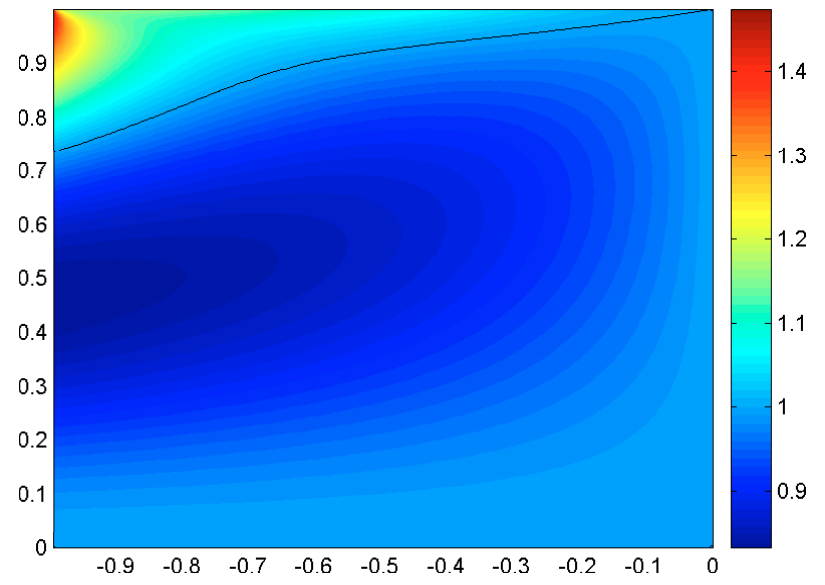

Figure 1 . The values of $L$ with respect to $\rho$ and $\mu$ with the level set $L=1$.

Proof of Theorem 1. Collecting the upper bounds on $A, B$ and $C$, we get $\|D K(0) \cdot h\|_{H^{1}}^{2} \leq L_{D K(0)}\|h\|_{H^{1}}^{2}$ with

$$
L_{D K(0)}=1-2 \theta \frac{1-\rho^{2}}{1-\tau \rho^{2}}+2\left[\theta \frac{1-\rho^{2}}{1-\tau \rho^{2}}\right]^{2}+\left(\frac{4}{5}+\frac{4}{3} \frac{1-\rho^{4}}{1-\tau}\right) \theta^{2} .
$$

Hence, the operator $D K(0)$ is a contraction if $L_{D K(0)}<1$. After simplification, this gives (31).

The condition (31) can be checked for each couple $(\rho, \mu)$. For example, one can check that if $\rho \leq 0.5$, then $D K(0)$ is a contraction. In Figure 1 we present a Matlab simulation of the constant $L_{D K(0)}$ in terms of $\rho$ and $\mu$. This shows that $D K(0)$ is a contraction for large radii, provided that the contrast $\mu$ of conductivities is sufficiently small. We recover the intuitive fact that for $\rho=1$, the method cannot converge since the problem is devoid of meaning: the inclusion fills the whole domain $\Omega$. By the same token, if $\mu=0$, then $L=1$; this fact corresponds to the case where the conductivities $\sigma_{1}$ and $\sigma_{2}$ are the same. One cannot hope to distinguish the inclusion $\omega$ in this situation. However, for small inclusions, the iterations converge.

From its expression (12), we see that $D K(0)$ depends continuously on $\psi$ and on the measure $(f, g)$. Since the solution of (1) depends continuously on $\partial \omega$, the continuity with respect to $(f, g)$ also means continuity with respect to the boundary $\partial \omega$. Hence, from Theorem 1, we deduce the following result:

Theorem 2. Assume that $\Omega \backslash \omega$ is close to an annulus bounded by concentric circles with an inner radius $\rho$ and that the pair of measures $(f, g)$ are closed to a sine function. If $\sigma_{2}>\sigma_{1}$ and (31), then the inclusion $\omega$ can be reconstructed via iterative approximations $\psi_{n+1}=K\left(\psi_{n}\right)$.

\subsection{Case of two measures}

We conclude this section by considering the case of two measures. For convenience, we also denote this second couple $(F, G)$. Note that $(F, G)$ are the traces of a solution to $(1)$ and that $(F, G)$ is chosen such that $g^{2}+G^{2} \geq \gamma$ holds for some $\gamma>0$. This leads to the fixed-point formulation (similar operators appear in [5]):

$$
\begin{aligned}
V: H^{1}(0,2 \pi) & \rightarrow L^{2}(0,2 \pi) \\
\psi & \mapsto \frac{(g \circ V \psi) \mathcal{D}_{\rho}[f \circ V \psi]+(G \circ V \psi) \mathcal{D}_{\rho}[F \circ V \psi]}{\left(g^{2}+G^{2}\right) \circ V \psi} . \\
Z: H^{1}(0,2 \pi) & \rightarrow H^{1}(0,2 \pi) \\
\psi & \mapsto \int_{0}^{t}\left(V \psi(x)-\frac{1}{2 \pi} \int_{0}^{2 \pi} V \psi(\tau) \mathrm{d} \tau\right) \mathrm{d} x .
\end{aligned}
$$


In this case, we obtain the contraction property without the restrictive condition (31). We linearize $Z$ in the neighborhood of 0 with the choice of measures made in (13) for $(f, g)$ and $F(t)=\cos t / \lambda_{1}(\rho)$ and $G(t)=\cos t$. We repeat the computations performed on $K$ to get

$$
D V(0) . h=\frac{\sin t}{\lambda_{1}(\rho)} \mathcal{D}_{\rho}[h(t) \cos (t)]-\frac{\cos t}{\lambda_{1}(\rho)} \mathcal{D}_{\rho}[h(t) \sin (t)]
$$

When we make this explicit on the basis $\left(\mathbf{C}_{k}, \mathbf{S}_{k}\right)$, we obtain only the diagonal term:

$$
\begin{gathered}
D Z(0) .\left[\begin{array}{l}
\mathbf{C}_{1} \\
\mathbf{S}_{1}
\end{array}\right]=\frac{\lambda_{2}(\rho)}{2 \lambda_{1}(\rho)}\left[\begin{array}{c}
\mathbf{C}_{1} \\
\mathbf{S}_{1}
\end{array}\right] \\
\forall k>1, D Z(0) .\left[\begin{array}{l}
\mathbf{C}_{k} \\
\mathbf{S}_{k}
\end{array}\right]=\frac{\lambda_{k+1}(\rho)-\lambda_{k-1}(\rho)}{2 k \lambda_{1}(\rho)}\left[\begin{array}{l}
\mathbf{C}_{k} \\
\mathbf{S}_{k}
\end{array}\right] .
\end{gathered}
$$

Notice that we still have to assume that $\sigma_{2}>\sigma_{1}$, since from (35), the first eigenvalue of $D Z(0)$ satisfies

$$
\frac{\lambda_{2}(\rho)}{2 \lambda_{1}(\rho)}<1 \Longrightarrow \mu<0 .
$$

Using the spectral gap bound (2), we obtain

$$
\forall k>1, \quad \frac{\lambda_{k+1}(\rho)-\lambda_{k-1}(\rho)}{2 k \lambda_{1}(\rho)}<\frac{\lambda_{2}(\rho)}{2 \lambda_{1}(\rho)}<1 .
$$

Hence, $D Z(0)$ is a contraction. This proves the following theorem for the two measures case.

Theorem 3 (the case of two measurements). Assume that $\Omega \backslash \omega$ is close to an annulus bounded by concentric circles, and that the pairs of measures $(f, g)$ and $(F, G)$ are closed to cosine and sine functions. If $\sigma_{2}>\sigma_{1}$, then the inclusion $\omega$ can be reconstructed via $\psi_{n+1}=K\left(\psi_{n}\right)$.

We should like to point out that this result is a direct generalization of Kress and Haddar's result, already proved in the perfectly insulating case in [5].

\section{Numerical EXPERIMENTS}

We present some numerical experiments involving the iterative methods presented in the previous sections. We should point out that our numerical simulation is not a real experiment. The synthetic data were obtained with the help of a forward solver providing output data. Furthermore, to avoid committing inverse crimes, the number of collocation points needed to obtain the current $g=\sigma_{1} \frac{\partial u}{\partial \mu}$ must be different from the number of discretization points within our location search method. All the numerical simulations were performed with the Dirichlet boundary data $f(t)=\sin t, t \in\left[0,2 \pi\right.$ [ and with a measured current $g_{m}$ which is obtained after adding random noise to the current. Since the resulting current on $\partial \Omega$ can be detected only at the attached electrodes, we assume that the measures $g_{m}$ are located at $M_{i}$ equidistant points on the circle $\partial \Omega$. In the following tests, the conductivities are taken as $\sigma_{1}=1$ and $\sigma_{2}=3$.

Circular inclusions: comparison of the methods

In the following example we try to recover the disk of center $\left(x_{c}=0.2, y_{c}=0\right)$ and radius 0.3 . The algorithm based on the fixed point is referred to as method 1. It has two steps:

(1) First determine the function $\psi$ and the inner radius $\rho^{*}$. This is done by projecting $\psi$ on the subspace of trigonometric polynomials of degree $N$. The choice of $N$ is for the moment arbitrary.

(2) Next, reconstruct the inner boundary, by attempting (via a least-square cost function) to identify the coefficients defining the Möbius transform. 


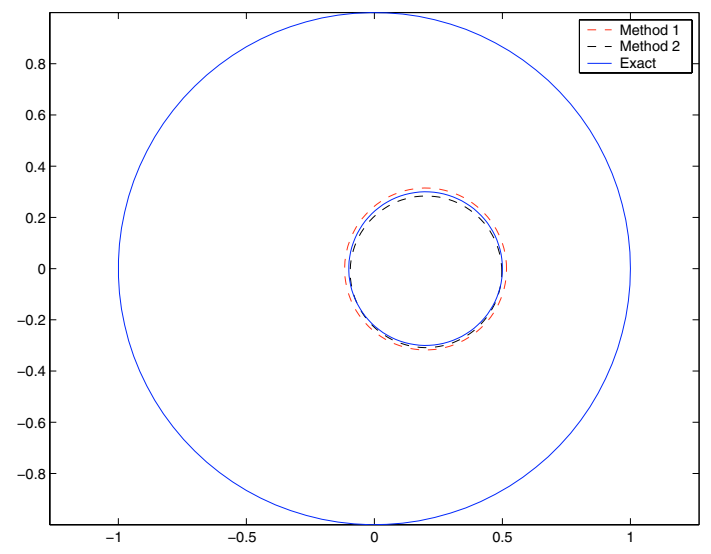

Exact parameters :

center : $(0.2,0), \quad$ radius : 0.3 ;

Reconstructed parameters :

Method 1 :

center : $(0.1999,-0.0013)$, radius : 0.3161 ;

Method 2 :

center : $(0.2016,-0.0125)$, radius : 0.2961 .

FIGURE 2. Reconstruction of a circular inclusion, no noise.
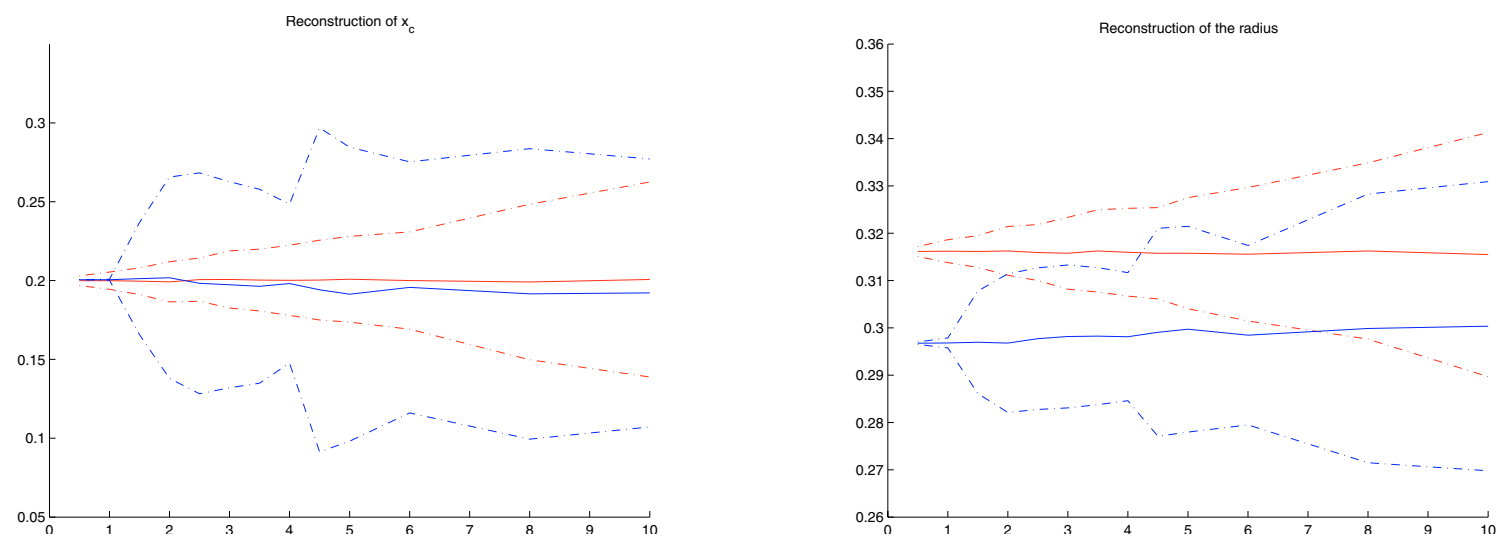

FIGURE 3. Reconstruction of a circular inclusion with respect to the noise level (in \%).

We refer to the algorithm based on the least-square solution of the integro-differential equation as method 2. The numerical results are presented in Figure 2.

Judging by these first results, there is little to choose between the two methods. The computational costs are of the same order. Let us consider the error for noised data. For each level of uniform noise added to the simulated Neumann data, 400 numerical experiments are performed. In order to make a fair comparison of the methods, we used identical parameters (and the same routines) for the optimization part of both algorithms. With method 2, this choice of parameters entails a frequent non-convergence of the optimization routine, so we end up with a much smaller size for the results sample, and the deviation from the mean is not really significant. Figure 3 presents the mean value, as well as an approximated confidence interval with bandwidth equal to three times the deviation from the mean. We observe that the method derived in Section 3 is more resistant to noise than the more elementary method introduced in Section 2.

\section{Extension of the conformal map}

This is the method used by Kress et al. $[1,5]$. Once we have found $\phi$ and $\rho$, we reconstruct the conformal transform $\Phi$. The inner boundary - that is to say our goal - is reconstructed as the image of the circle whose center is the origin, and whose radius is $\rho$, by the holomorphic extension $\Phi$ of $\phi$. From a numerical point of view, this requires the conformal mapping $\Phi$ to be determined. This problem, in Hadamard's terminology, is 

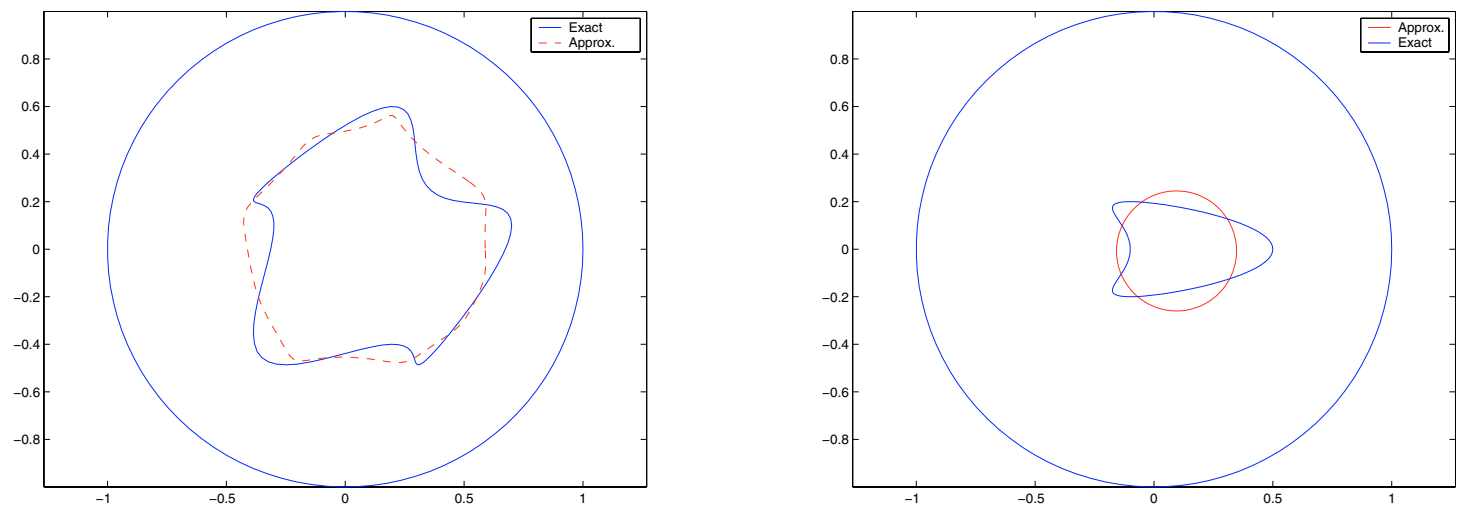

FIGURE 4. Reconstruction of a kit-shaped inclusion: numerical extension and approximated circle.

not well-posed. A typical approximation of $\phi$ is obtained via truncated Fourier series :

$$
\phi(t) \approx \sum_{k=1}^{N} a_{k} \cos k t+b_{k} \sin k t=\sum_{k=1}^{N} \frac{a_{k}-i b_{k}}{2} \mathrm{e}^{i k t}+\sum_{k=1}^{N} \frac{a_{k}+i b_{k}}{2} \mathrm{e}^{-i k t} .
$$

Then $\Phi$ can be expressed

$$
\Phi\left(r \mathrm{e}^{i t}\right) \approx \sum_{k=1}^{N} \frac{a_{k}-i b_{k}}{2} r^{k} \mathrm{e}^{i k t}+\frac{a_{k}+i b_{k}}{2} r^{-k} \mathrm{e}^{-i k t}
$$

The numerical instability appears in the negative powers of $r$ : any error in the numerical approximation of the Fourier coefficients is amplified by the exponential factor $r^{-k}$. This fact is well-known, and to avoid instabilities we need to regularize using a Tykhonov penalization. This means adding some regularization parameters $\varepsilon_{k}$ chosen by the discrepancy principle. Hence, we use the regularized conformal mapping $\Phi_{r}$ :

$$
\Phi_{r}\left(r \mathrm{e}^{i t}\right) \approx \sum_{k=1}^{N} \frac{a_{k}-i b_{k}}{2} r^{k} \mathrm{e}^{i k t}+\frac{a_{k}+i b_{k}}{2} \frac{r^{k}}{\varepsilon_{k}+r^{2 k}} \mathrm{e}^{-i k t}
$$

The shape of the inclusion is then recovered as $\omega_{r} \approx \Phi_{r}\left(\rho \mathrm{e}^{i \theta}\right)$.

This way of constructing a conformal extension is not limited to Möbius transforms. We tested this way of extending the function obtained after the first step of method 1 by applying it to noncircular inclusions. The numerical results are presented in Figure 4. They show that the fixed-point-based method proposed in this work can identify inclusions that are not circular. In [2] we shall be providing a theoretical explanation.

In the case of noncircular inclusions, our method gives a good circular approximation. The theoretical basis for this is the notion of approximate identifiability, introduced in [3] by Fabes et al. In their paper they consider two domains $D_{0}$ and $D_{1}$ belonging to $\mathcal{C}(\varepsilon)$, the class of $\varepsilon$ perturbations of all disks contained in $\Omega_{0}$, an open subset of $\Omega$ at some distance, say $2 \delta_{0}$, from $\partial \Omega$. They prove the following important result: There exists a positive constant $C>0$ such that if $\Lambda_{D}(g)=\Lambda_{D_{0}}(g)=f$ then $\left|D \Delta D_{0}\right| \leq C \varepsilon$.

Acknowledgements. Our thanks are due to Pr. Kress and to the anonymous referee for their helpful comments. 


\section{REFERENCES}

[1] I. Akduman and R. Kress, Electrostatic imaging via conformal mapping, Inverse Problems 18 (2002) 1659-1672.

[2] M. Dambrine and D. Kateb, Work in progress.

[3] E. Fabes, H. Kang and J.K. Seo, Inverse conductivity problem with one measurement: Error estimates and approximate identification for perturbed disks. SIAM J. Math. Anal. 30 (1999) 699-720.

[4] G.M. Golutsin, Geometrische Funktionentheorie. Deutscher Verlag der Wissenschaften, Berlin (1957).

[5] H. Haddar and R. Kress, Conformal mappings and inverse boundary value problems. Inverse Problems 21 (2005) 935-953.

[6] P. Henrici, Applied and computational complex analysis, Vol 1,3. John Wiley \& Sons (1986).

[7] N.I. Muskhelishvili, Some basic problems of the mathematical theory of elasticity. Noordhoff, Groniningen (1953).

[8] M. Taylor, Partial Differential Equations, Vol. 1: Basic Theory. Applied Math. Sciences 115, Springer-Verlag, New York (1996). 